\title{
Reducing the severity of a traffic accident
}

\author{
Sergey Dorokhin ${ }^{1, *}$, Valery Ivannikov ${ }^{1}$, Konstantin Yakovlev $^{1}$, Andrey Shvyriov ${ }^{1}$, \\ Alexander Shemyakin ${ }^{2}$, Sergey Borychev ${ }^{2}$, and Alexey Terentyev ${ }^{3}$ \\ ${ }^{1}$ Voronezh State University of Forestry and Technologies named after G. F. Morozov, 8, Timiryazeva \\ str., 394087, Voronezh, Russia \\ ${ }^{2}$ Ryazan State Agrotechnological University Named After P.A. Kostychev, 1, Kostychev Str., \\ 390044, Ryazan, Russia \\ ${ }^{3}$ Academy of Law and Management of the Federal Service for the Execution of Sanctions, 1, Sennaya \\ Str., 390000, Ryazan, Russia
}

\begin{abstract}
Improving the system of preventive measures aimed at reducing the severity of road accidents is an urgent task. Mortality on roads is constantly increasing and it is necessary to ensure an integrated approach to creating safe road conditions. The purpose of this study is to analyze the promising designs of guardrails designed to prevent uncontrolled exit of vehicles from the roadway and the development of crash cushions. Guardrails should not only be safe for road users, but should also ensure their safety, as well as preserve the elements after crashing it. Conducted analytical studies have shown that to reduce the mechanical damage to vehicles and reduce the severity of injuries to the driver and passengers, it is necessary to develop guardrails that allows capturing shock energy at the moment of contact between the car and the guardrails. The considered design of the crash cushion provides a damping effect when the car crashes the guardrails and at the same time limit the ability to move its elements relative to the original position. This is achieved by using several materials with different strength characteristics in the design of the guardrails, which contributes to its gradual destruction in several stages and smooth energy dissipation.
\end{abstract}

\section{Introduction}

An objective assessment of the level of traffic safety on roads indicates that constantly increasing traffic causes a large number of conflict situations and, as a result, has led to an increase in the number of road traffic accidents (RTAs). Driving on modern highways requires drivers to have a high concentration of attention while driving and constantly improving their professional skills, as well as strict observance of traffic rules. At present, it is obvious that it is not possible to change the situation with the growth of road injuries only by making decisions at the legislative level. The increase in the number of administrative sanctions should be considered only as a preventive measure (in most cases, it is a punishment for an already committed violation of traffic rules), often acting only in the presence of traffic police officers or in the area of installed video recording cameras.

\footnotetext{
*Corresponding author: kosta066@yandex.ru
} 
The number of fatal accidents and serious injuries sustained as a result of accidents can be reduced through the use of an integrated approach to road safety. Strategies and programs for improving road traffic should include the following measures: reducing the risk of road traffic accidents, preventing accidents, reducing the number of injuries from road traffic accidents, and reducing the consequences of injuries by improving health care after road traffic accidents [1,2].

A report by the World Health Organization (WHO) indicates that road traffic deaths continue to increase, amounting to 1.35 million deaths per year, and injuries sustained in car accidents are the eighth leading cause of death [3]. At the same time, the risk of death due to road traffic accidents in low-income countries is still three times higher than in highincome countries. The highest rates are observed in Africa (26.6 cases per 100,000 people), and the lowest are observed in Europe (9.3 cases per 100,000 people). According to official statistics in the Russian Federation in 2018 there were more than 168 thousand accidents, in which 18,214 people died and 214,853 people received injuries of varying severity [4]. These sad statistics require the development of modern ways to prevent accidents on roads. Legislative decisions in a number of countries have been made to reduce road traffic injuries. The main goal of such decisions is "Vision Zero", i.e. the number of fatal accidents should be reduced to zero in the long term. In January 2018, the Government of the Russian Federation approved the Road Safety Strategy in the Russian Federation for 2018-2024, which stated that one of the main directions of its implementation is to improve the road network in terms of road safety, including the development of road traffic management $[5,6]$.

According to statistics, approximately every fourth accident on the road is caused by an unintentional (uncontrolled) exit of cars from the roadway, which is characterized by serious damage to vehicles, death and personal injury, as well as material damage of transported goods. One of the ways to prevent accidents is the installation of various guardrails on the road. Guardrails allow keeping the car on the roadway, but at the same time they receive mechanical damage, and since damping is mainly due to deformation of the body, this is extremely dangerous for passengers who experience powerful overloads. At the same time, a deformed body can create additional difficulties in evacuating the driver and passengers from the damaged car. Given the high likelihood of serious injury to people and serious mechanical damage to the vehicle when hitting an obstacle (guardrail), it is necessary that if the car goes out of the road, it crashes into the guardrail and does not return to the lane and damage to it were minimal [7]. In our work, we will analyze the structures of modern guardrails and consider the possibility of using a crash cushion that reduces the severity of the consequences of an accident.

\section{Discussion}

Constantly increasing traffic and permissible speed limits impose more stringent requirements on road infrastructure and the use of road safety systems. One way to improve road safety is to use road restraint systems. Guardrails do not only reduce the number of road traffic accidents, but also reduce their severity [8]. Considerable attention is paid to the choice of guardrails for the safe operation of roads, bridge structures and the safety of road users: drivers and passengers of motor vehicles, non-motorized and horse-drawn vehicles, pedestrians, livestock and wild cattle. Guardrails are considered a simple but at the same time very important element of the road infrastructure. Thanks to the knowledge about their design, it is possible to reduce the number of accidents on the road significantly, thereby preventing a possible accident and minimizing damage in it, if any. One of the most important characteristics of a guardrail is its holding capacity (energy intensity), i.e. the ability of the guardrail to keep vehicles on the road and bridge structure, while not allowing 
them to tip over or move over it. The holding ability is divided into levels, each of which has its own range of striking energy. It is determined depending on the category of road, permissible speed and groups of road conditions [9].

Today, there are many scientific works by both foreign and domestic authors on the topic of guardrails. The study of the design of domestic and foreign guardrails was carried out by Badoyan N.Sh., Schepetova L.S. and Pugin K.G. [10]. The authors presented a detailed analysis of guardrails used in highway construction in many countries of the world. The paper considers the theoretical and empirical aspects of guardrails. An analysis of traditional guardrails and new foreign developments is provided. A new type of gabion barrier, which has not practically been considered by any of researchers as a tool to protect against traffic accidents on roads, is presented. To conduct an empirical assessment of benefits of gabions in comparison with classical guardrails, the article presents a model, as well as software packages with which it is possible to evaluate the effectiveness of gabions as safety barriers. A study and experience of road-bridge construction in a number of countries, including domestic experience of recent years, showed that gabion structures have very broad capabilities and properties, such as efficiency, strength and durability, which are the key ones in highway construction. British scientists G. Amato, F. O'Brien, B. Ghosh and C. Simms [11] evaluated the potential of gabions as safety barriers on roads, should also be emphasized. To achieve this goal, the authors created a prototype of the gabion safety barrier, which during the study was subjected to total refinement and testing using crash tests in accordance with European standards EN1317 for protective barriers N1. Thanks to the crash tests performed in the empirical part of the work, the authors revealed that a collision of a car and a gabion safety barrier led to a rollover of the vehicle and a break in the gabion net. Unlike classic guardrails, gabion structures have many advantages, thanks to which it is possible to reduce the risk of accidents, as well as the severity of consequences for humans [12].

French scientists have conducted studies to assess the impact of longitudinal guardrails located on the middle lanes and hard shoulders of toll roads on the severity of an accident with vehicles moving off the road. The study was based on accidents related only to injuries and property damage, recorded over 15 years on the French network of toll motorways with a length of about $2,000 \mathrm{~km}$. When leaving the roadway to a solid shoulder, the risk of injury was halved by the longitudinal guardrail. The specific one-sided W-radial guardrail ("GS4") proved to be the best solution for cars, buses and trucks. This does not affect the feasibility of special guardrails for bridges or concrete barriers when a narrow working width is required. Longitudinal guardrails are important for the safety of road users, providing "forgiving" infrastructure in the event a vehicle exits the road, provided there are very few motorized two-wheeled vehicles on the roadway $[13,14]$.

Employees of Orenburg State University conducted research on a safe guardrail called Road Roller System, being a new type of road safety system that disperses the force of impact. Instead of a sturdy metal beam, this design uses many rollers. When the car enters the "roller guardrail", even at a right angle, they redirect the impact force of the car, turning that energy, which could be fatal, into a much less powerful one. Technically, the Road Roller System consists of sturdy steel pipes, between which there are plastic rollers rotating around its axis. Rollers are bright yellow with reflective stripes. When a car collides, the guardrail bends and works like a shock absorber, taking on most of the shock, and the rollers rotate, due to which the car's inertia is suppressed and the emergency trajectory changes (turn the car sideways to the guardrail and smoothly return it to the road). As a result, the nature and amount of damage to the car, as well as injuries of people are reduced, and the likelihood of a rollover is minimized [15].

Australian scientists [16] conducted studied the response to shock of a portable waterfilled barrier (PWFB), which has the potential to absorb impact energy and, therefore, 
mitigate the effects of the accident at low and moderate speeds. Modern studies of the shock and energy absorption capacity of water-filled barriers are limited due to the complexity of the interaction of the fluid structure under dynamic impact. In the present work, a new method for the interaction of a fluid and a structure is developed, based on a combination of the hydrodynamics of smooth particles and the finite element method. The phenomenon of water splash inside the PWFB was investigated to study the ability of water to absorb the energy under dynamic exposure. It was found that water plays an important role in energy absorption. The link analysis presented in this article provides a platform for further research to optimize the PWFB. The effect of the amount of water on its energycapturing ability was studied and the results found practical application in the design of the PWFB.

To reduce the severity of the consequences of an accident, the authors of [17] propose using torsion energy-capturing elements in guardrails, the principle of which is based on the dissipation of impact energy due to plastic torsion of metal rods. The choice of torsion elements is based on the combination of their positive qualities. Torsion energy-capturing elements have a specific energy consumption that exceeds the similar indicators of known shock absorbers. They can be placed in narrow gaps, are very technologically advanced to manufacture and easy to operate. Their power characteristic is practically independent of the speed of exposure and environmental parameters. In addition, the torsion energycapturing element has another positive quality, namely that a partially or fully deformed element can be repeatedly brought back to its original position, and its energy-capturing ability is restored.

\section{Energy-capturing guardrail}

The analysis makes possible to formulate a number of requirements for modern designs of guardrails. Guardrails should not only be safe for road users, but should also preserve the elements after hitting the guardrail. Guardrails can be considered safe if in a case when the vehicle contacts them [7]:

- guardrail elements did not get into the cabin;

- the car did not turn over, did not damage it and did not turn around after the collision;

- an overload per person and deformation of the car cab do not lead to serious injuries.

Besides, the design of the developed guardrails should be quite simple, and be able to be mounted either already on existing road barriers, or completely replace them. Moreover, the installation process should be extremely simple and very fast. It should also be borne in mind that this system is destructible, that is, it is necessary to take into account its minimum cost in the design.

All of the above circumstances allow to conclude that there is a need to develop a design of energy-capturing guardrails, which allows, if not completely eliminating undesirable consequences for road accident participants, then at least reducing them to the lowest possible level. Consider the basic requirements for materials for the manufacture of guardrails:

- high energy-capturing ability, i.e. the material should have sufficient porosity or low density, but at the same time it should be easily compacted when exposed to;

- the possibility of using various climatic conditions, i.e. the characteristics of such materials should slightly vary depending on the ambient temperature, humidity and sunlight intensity;

- the guardrail must be assembled from separate damping elements, united by flexible connections between each other in the section, which would provide the possibility of limited movement of the guardrail elements relative to their initial position in a case of hammering action by the car; 
- low cost of these materials.

Given the above requirements for materials, it can be assumed that a single material, will probably not be able to satisfy all the requirements at the same time, so we suggest using the so-called sandwich construction, i.e. the construction should consist of the following materials (Fig. 1):

- the structural or "skeleton" material, which will act as a supporting structure, and ensure the gradual destruction of the guardrail, from layer to layer;

- the energy-capturing material that will capture the impact energy due to compaction or destruction of the base layer;

- the external protective material, which should have increased abrasion resistance, have anti-corrosion properties and perform the function of a protective element.

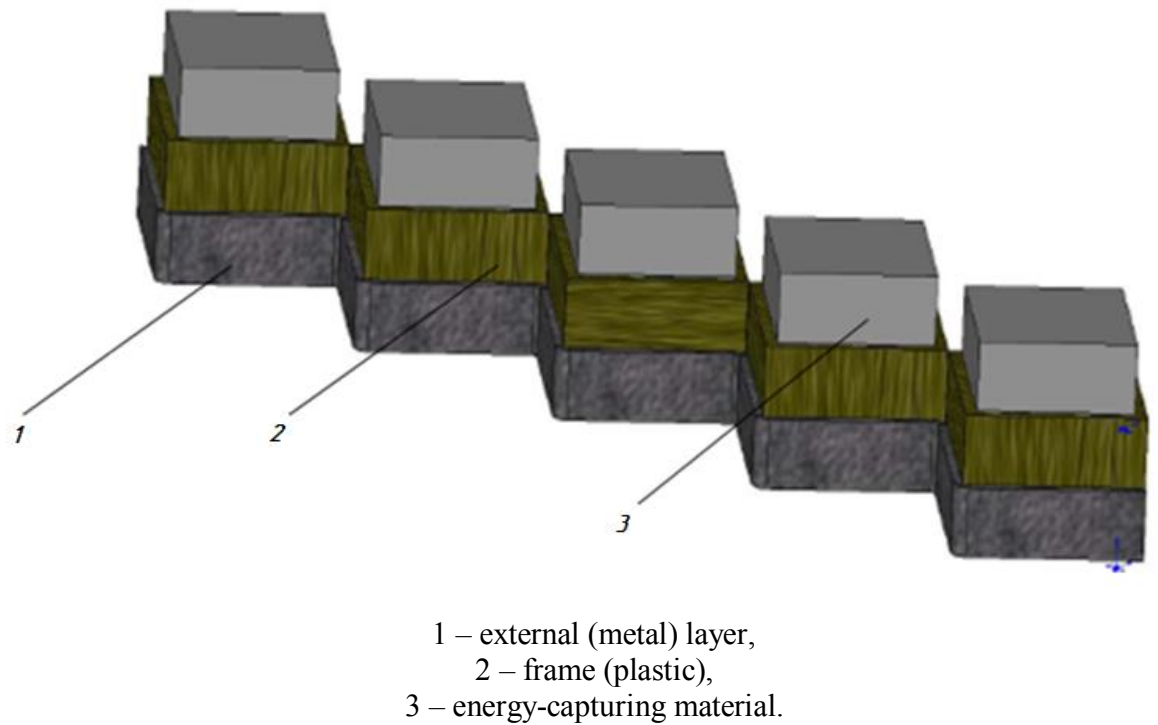

Fig. 1. Energy-capturing guardrail.

To give the design the required characteristics, it is necessary to determine the most promising materials that can be used in structures of this type. Let us consider them in the sequence in which they are shown in Fig. 1. The external protective layer must have the properties described above, therefore, it is proposed to use specially molded sheet metal with a galvanized surface or special plastic spraying for its manufacture. In this case, the profile of sheet metal, which is an undulated surface, can be obtained using conventional bending on a bending machine from a conventional, for example, galvanized sheet metal. The thickness of such a sheet should provide easy bending when forming a profile, but at the same time, have rigidity and strength sufficient to withstand external influences unrelated to the suppression of collision energy. It should be borne in mind that the thickness of the sheet of metal should not exceed the thickness of the external body elements of the car, i.e. when a car collides with a guardrail, its outer layer must first deform, and then the body panels of the car. Based on these assumptions and according to the Dassault System databases, the most suitable option is to use a galvanized metal with a thickness of $0.25 \mathrm{~mm}$.

Next, it is necessary to determine the material that can be used to create a power structure, the so-called skeleton of an energy-capturing guardrail. For these purposes, it is most rational to use one of the types of plastics. The so-called PVC plastic, being one of the most economical options, should be of prime attention. This material is well formed, there 
are many enterprises that are engaged in its industrial processing. In particular, water-filled barriers are made of this material. The production base is debugged. In addition, only molds will be required. The general view of a single cell, made according to the principle of combs, may have the view presented in Fig. 2.

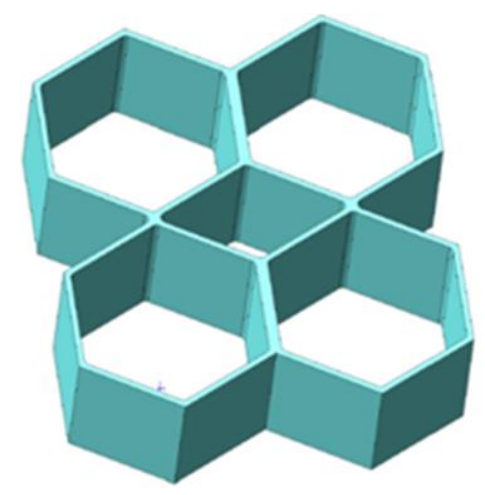

Fig. 2. The structure of the "skeleton" part.

The energy-capturing material should have the properties of low density and hygroscopicity, as well as not have an elastic component of deformations, so it is most rational to use fibrous materials for its manufacture, for example, one of the types of Thermoisol. The general view of the structure of the proposed guardrail will have the form presented in Fig. 3 [7].

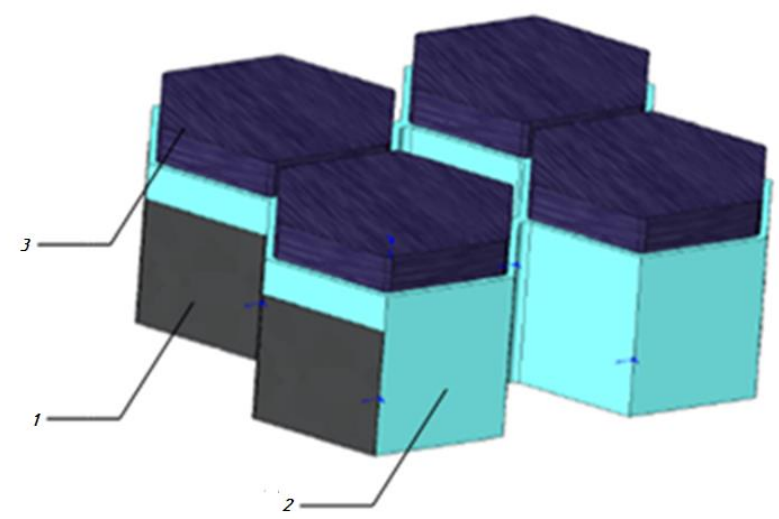

1 - profiled steel galvanized sheet (thickness $0.25 \mathrm{~mm}$ ); 2 - "skeleton" of the guardrail (hard PVC); 3 - energy-capturing material (Thermoisol 900)

Fig. 3. General view of a single cell.

\section{Conclusion}

Improving road safety by introducing administrative restrictions and imposing penalties for violation of traffic rules is almost impossible to achieve. Consequently, an integrated approach to solving this problem is required involving representatives of the legislative branch, specialists engaged in practical activities in the road industry, as well as research and development organizations.

Preventing RTAs, as well as reducing the severity of their consequences, is possible through the installation of guardrails for various purposes and design. The analysis showed 
that at present there is a wide range of various construction guardrails, the use of which makes it possible to keep the vehicle on the road, but it does not always take into account the severity of the consequences of the interaction of the car and the guardrails.

The design of the energy-capturing guardrail considered in this work allows to reduce the likelihood of severe injuries of road users in a case of an uncontrolled vehicle exit from the roadway. The damping properties of this guardrail provide a smooth damping of impact energy when a car hits it and thereby reduce the loads experienced by road accident participants. Unlike most analogues, the design of energy-capturing guardrail during shock contact with the vehicle makes possible to limit the ability to move its elements relative to the original position.

\section{References}

1. K. Goniewicz, M. Goniewicz, W. Pawłowski, P. Fiedor, European Journal of Trauma and Emergency Surgery 42(4) (2015) DOI: 10.1007/s00068-015-0544-6

2. K. Andreev, V. Terentyev, E3S 135, 02013 (2019) DOI: $10.1051 / \mathrm{e} 3$ sconf $/ 201913502013$

3. https://www.who.int/violence_injury_prevention/road_safety_status/2018/Pressrelease-GSRRS2018-RU.pdf?ua=1

4. https://gibdd.rf/

5. Road Safety Strategy in the Russian Federation for 2018-2024 (Government of the Russian Federation, 2018)

6. V. Zelikov, G. Denisov, S. DorokhinStudies in Computational Intelligence 826, 10811088 (2019) DOI: 10.1007/978-3-030-13397-9_111

7. K. Andreev, V. Terentyev, A. Shemyakin, Transport facilities. Ecology 1, 5-12 (2018) DOI: $10.15593 / 24111678 / 2018.01 .01$

8. Ž. Butāns, K. Gross, A. Gridnevs, E. Karzubova, IOP Conf. Series: Materials Science and Engineering 96, 012063 (2015) DOI:10.1088/1757-899X/96/1/012063

9. A. Trapeznikov, R. Borisov, D. Shcherbakov, V. Koshelev, D. Churkin, A. Mordvinov, N. Bogoiavlenskii, Ecology 4, 13-25 (2019) DOI 10.15593/24111678/ 2019.04.02

10. N. Badoyan, L. Shepeteva, K. Pugin, Russian journal of transport engineering 2(6) (2019) DOI: 10.15862/05SATS219

11. G. Amato, F. O'Brien, B. Ghosh, C. Simms, International Journal of Crashworthiness 20(1), 12-26 (2015) DOI: 10.1080/13588265.2014.949038

12. G. Amato, F. O'Brien, C. Simms, B. Ghosh, International Journal of Crashworthiness 18, 237-250 (2013) DOI: 10.1080/13588265.2013.775739

13. J. Martin, C. Mintsa-Eya, C. Goubel, Accident Analysis and Prevention 59, 443-451 (2013) DOI: 10.1016/j.aap.2013.06.024

14. M. Rencelj, T. Tollazzi, S. Turnsek, M. Sraml, Procedia - Social and Behavioral Sciences 53, 646-655 (2012) DOI: 10.1016/j.sbspro.2012.09.914

15. S. Dergunov, S. Orehov, E. Taranovskaya, N. Samigullin, Trends in the development of science and education 26(4), 69-71 (2017) DOI: 10.18411/lj-31-05-2017-72

16. I. Thiyahuddin, Y. Gu, D. Thambiratnam, P. Gudimetla, International Journal of Protective Structures 3(3), 257-273 (2012) DOI: 10.1260/2041-4196.3.3.257

17. A. Reshenkin, V. Tikhomirov, ISJ Theoretical \& Applied Science 10(30), 105-110 (2015) DOI: $10.15863 /$ TAS.2015.10.30.23 